\title{
Degradation of Cellulosic Materials under the Alkaline Conditions of a Cementitious Repository for Low- and Intermediate Level Radioactive Waste. Part III: Effect of Degradation Products on the Sorption of Radionuclides on Feldspar
}

\author{
By L. R. Van Loon*, M. A. Glaus, A. Laube and S. Stallone \\ Paul Scherrer Institute, Laboratory for Waste Management, CH-5232 Villigen PSI, Switzerland
}

(Received April 14, 1999; accepted in revised form July 7, 1999)

\section{Cellulose / Isosaccharinic acid / Complexation / Sorption / Radionuclides}

\begin{abstract}
Summary
The effect of degradation products of different cellulosic materials on the sorption behaviour of Th(IV), Eu(III) and Ni(II) on feldspar at pH 13.3 was studied. For all three metals, a decrease in sorption could be observed with increasing concentration of organics in solution. For Th(IV), $\alpha$-ISA is the effective ligand present in the solutions of degraded cellulose, independent on the type of cellulose studied. For Eu(III), $\alpha$-ISA is the effective ligand in the case of pure cellulose degradation. In the case of other cellulosic materials, unknown ligands cause the sorption reduction. For $\mathrm{Ni}(\mathrm{II})$, also unknown ligands cause sorption reduction, independent on the type of cellulose studied. These unknown ligands are not formed during alkaline degradation of cellulose, but are present as impurities in certain cellulosic materials.
\end{abstract}

\section{Introduction}

The degradation of cellulose in a cementitious repository for radioactive waste results in the formation of water soluble organics. It was recently shown that isosaccharinic acid (ISA) is the main degradation product [1-3], contributing up to $\sim 80 \%$ of the total. Two diastereoisomers, $\alpha$ - and $\beta$-ISA, are formed in equal amounts $[1,2]$. ISA belongs to the polyhydroxy ligands and - by analogy with gluconic acid [4] - is assumed to form strong complexes with tri- and tetravalent metals, especially under alkaline conditions. The complexation reactions are not yet understood in detail, but it seems that under alkaline conditions hydroxylic groups are involved in the coordination reaction $[5,6]$. Complexation of radionuclides by (organic) ligands can affect their mobility in the near- and the far-fields of a radioactive waste repository. It has been shown that cellulose degradation products strongly increase the solubility of $\mathrm{Pu}(\mathrm{IV})$ [7, 8] and several other metals such as $\mathrm{Cu}$ (II), $\mathrm{Co}$ (II), Eu(III) and Sm(III) [9] under alkaline conditions. This increase was interpreted as being due to a strong complexation of these metals with polyhydroxy ligands,

\footnotetext{
* Author for correspondence

(E-mail: Luc.VANLOON@PSI.CH).
}

probably ISA [8, 9]. Degradation products of cellulose can also decrease the sorption of tri- and tetravalent metals on rock materials [10-12]. All studies previously reported used degradation products of cellulose obtained at elevated temperatures. Data for ambient temperature are not available. Further, although ISA is suspected to be responsible for the observed sorption reduction effects, there are no studies available showing quantitatively the correlation between the concentration of ISA present in solutions of degraded cellulose and sorption reduction caused by ISA.

This study describes experiments on the effect of cellulose degradation products on the sorption of $\mathrm{Ni}(\mathrm{II}), \mathrm{Eu}(\mathrm{III})$ and Th(IV). The degradation of different cellulose materials was studied at ambient temperature $\left(25 \pm 2{ }^{\circ} \mathrm{C}\right)$ and the observed sorption reduction was correlated with the concentration of the main degradation product present. For this purpose a model based on possible complexation reactions in solution is proposed to describe ligand-induced sorption reduction. The solid phase used in this study was a feldspar. Because ISA does not sorb on feldspar [13], the use of feldspar simplifies the ternary system in such a way that the sorption of the ligand and the sorption of complexes have not to be considered in the model, leaving complexation reactions in solution as the only relevant reactions.

\section{Materials and methods}

Chemicals

All chemicals used in this study were of analytical grade quality. The water used was demineralised water (Milli-Q water, Millipore, USA).

\section{Feldspar}

The feldspar used in the experiments was an orthoclase (Fronland, Norway). The feldspar was crushed and sieved. The fraction $<63 \mu \mathrm{m}$ was used in the sorption studies. 


\section{Cellulose degradation}

$100 \mathrm{~g}$ of cellulosic materials with varying degree of polymerisation (Aldrich cellulose, cotton, tissues and paper), were contacted with 11 of artificial cement pore water (ACW-I) having the following composition: $114 \mathrm{mmol} \cdot \mathrm{l}^{-1} \mathrm{Na}, 180 \mathrm{mmol} \cdot \mathrm{l}^{-1} \mathrm{~K}, 2 \mathrm{mmol} \cdot \mathrm{l}^{-1}$ $\mathrm{Ca}$, and $300 \mathrm{mmol} \cdot 1^{-1} \mathrm{OH}^{-}(\mathrm{pH} \sim 13.3)$. The ACW-I simulates approximately the cement pore water composition of the first stage of cement degradation [14]. The experiments were carried out in a glove box under a controlled $\mathrm{N}_{2}$ atmosphere $\left(\mathrm{CO}_{2}, \mathrm{O}_{2}<5 \mathrm{ppm}\right)$ at a temperature of $25 \pm 2{ }^{\circ} \mathrm{C}$. A detailed description of the cellulose degradation and information on the cellulosic materials used, is given elsewhere $[1,2]$. At given time intervals, samples of the aqueous phase containing the degradation products were filtered ( $\mathrm{Te}$ flon membrane filter, type FH, $0.5 \mu \mathrm{m}$, Millipore) and analysed for isosaccharinic acid (Dionex Carbopac PA-100; $250 \times 5 \mathrm{~mm}$ column, $\mathrm{T}=20^{\circ} \mathrm{C}$, flow rate: $1 \mathrm{ml} \cdot \mathrm{min}^{-1}$, eluent: $0.1 \mathrm{M} \mathrm{NaOH}+$ gradient of $\mathrm{NaOAc}$; pulsed amperometric detection with a gold working electrode [15]).

\section{Solutions for sorption experiments}

The degradation products, obtained at different degradation times, were used both undiluted and diluted in the sorption experiments. The dilutions were made by putting $100 \mathrm{ml}, 10 \mathrm{ml}$ or $1 \mathrm{ml}$ of the degradation products into a $100 \mathrm{ml}$ flask. The volume was made up to $100 \mathrm{ml}$ by adding ACW-I. Standard solutions of $\alpha$-ISA and $\beta$-ISA were prepared in ACW-I in a concentration range between $10^{-6}$ and $10^{-1} \mathrm{M}$. The $\alpha$-ISA solutions were made from $\mathrm{Na}(\alpha$-ISA) which was synthesised as described in [1]. $\beta$-ISA was isolated in an almost pure form from a mixture of cellulose degradation products as described in [1].

\section{Sorption experiments with Eu(III)}

$20 \mathrm{mg}$ of feldspar were placed in $50 \mathrm{ml}$ polyallomer centrifuge tubes and contacted with $2 \mathrm{ml}$ of ACW-I containing ${ }^{152} \mathrm{Eu}\left(4.44 \mathrm{kBq} \cdot \mathrm{ml}^{-1}\right)$. After 2 hours, $28 \mathrm{ml}$ of either ACW-I, $\mathrm{Na}(\alpha$-ISA) or degradation solutions were added ${ }^{1}$. The total concentration of Eu added to the suspensions was approximately $6.7 \cdot 10^{-10} \mathrm{M}$. The suspensions were further equilibrated for $24 \mathrm{~h}$. This time was found to be sufficient to reach nearly equilibrium. A $1 \mathrm{ml}$ sample of the homogenised suspensions was taken without prior centrifuging and analysed for ${ }^{152} \mathrm{Eu}$ by $\gamma$-counting (Minaxi- $\gamma$, autogamma ${ }^{\circledR 5} 500$ series, Packard). The rest of the suspension was centrifuged for 15 minutes at 27,000

\footnotetext{
1 Preliminary experiments showed that, when the Eu-ISA or ThISA complex was added to the solid phase, equilibrium was reached only after $72 \mathrm{~h}$. Adding the free metal first, followed by adding the ligand resulted in constant sorption values after $2 \mathrm{~h}$. The same equilibrium value for $K_{d}$, however, was obtained independent of the way the nuclides were added.
}

g (L7-35 ultracentrifuge, Beckman), the supernatant solution sampled $(2 \mathrm{ml})$ and analysed for ${ }^{152} \mathrm{Eu}$. The measurements without prior centrifuging $\left(\mathrm{A}_{\text {susp }}\right)$ give information on the total concentration of ${ }^{152} \mathrm{Eu}$ in the suspension (sum of ${ }^{152} \mathrm{Eu}$ on the solid phase and ${ }^{152} \mathrm{Eu}$ in the liquid phase). Measurements of the supernatant solution $\left(\mathrm{A}_{\mathrm{eq}}\right)$ give information on the ${ }^{152} \mathrm{Eu}$ in the equilibrium solutions only. The distribution ratio $\left(\mathrm{K}_{\mathrm{d}}\right.$, $1 \cdot \mathrm{kg}^{-1}$ ) was calculated by the difference in radioactivity in solution before and after centrifugation:

$$
\mathrm{K}_{\mathrm{d}}=\frac{\left(\mathrm{A}_{\mathrm{susp}}-\mathrm{A}_{\mathrm{eq}}\right)}{\mathrm{A}_{\mathrm{eq}}} \cdot \frac{\mathrm{V}}{\mathrm{W}}
$$

where

$\mathrm{A}_{\text {susp }}=$ the radioactivity of ${ }^{152} \mathrm{Eu}$ in the suspension $\left(\mathrm{cpm} \cdot \mathrm{1}^{-1}\right)$

$\mathrm{A}_{\mathrm{eq}}=$ the radioactivity of ${ }^{152} \mathrm{Eu}$ in the supernatant solution $\left(\mathrm{cpm} \cdot \mathrm{1}^{-1}\right)$

$\mathrm{V}=$ volume of the suspension (l)

$\mathrm{W}=$ mass of the solid phase $(\mathrm{kg})$.

Due to the restricted amount of $\beta$-ISA available in pure form, the sorption experiments were conducted at reduced volumes. Preliminary tests with $\alpha$-ISA showed that it was possible to conduct sorption experiments in a volume of $2 \mathrm{ml}$. For practical reasons these sorption experiments were not conducted in a glove box under a controlled $\mathrm{N}_{2}$ atmosphere. $100 \mu \mathrm{l}$ of a feldspar suspension ( $660 \mathrm{mg}$ feldspar $+20 \mathrm{ml}$ ACW-I) were contacted with $100 \mu \mathrm{l}$ of an ${ }^{152} \mathrm{Eu}$ spiked solution $\left(20 \mathrm{ml} \mathrm{ACW}-\mathrm{I}+80 \mu \mathrm{l}{ }^{152} \mathrm{Eu}\right.$ stock solution) for $1 \mathrm{~h}$. The concentration of Eu added was $6.7 \cdot 10^{-10} \mathrm{M}$. Aliquots of a $\beta$-ISA solution were added. After $24 \mathrm{~h}$ contact time - the vials were shaken by hand from time to time - the suspensions were filtered by a $0.45 \mu \mathrm{m}$ filter and the amount of ${ }^{152} \mathrm{Eu}$ in the equilibrium solution measured as described earlier. No loss of ${ }^{152} \mathrm{Eu}$ on the filter material was observed.

\section{Sorption experiments with Th(IV)}

$20 \mathrm{mg}$ of feldspar were placed in $50 \mathrm{ml}$ centrifuge vials and contacted with $2 \mathrm{ml}$ of ACW-I containing ${ }^{234} \mathrm{Th} /{ }^{332} \mathrm{Th}$. The carrier-free ${ }^{234} \mathrm{Th}$ was prepared from ${ }^{238} \mathrm{U}$ as described in Dyrssen [16]. After 2 hours, $28 \mathrm{ml}$ of either ACW-I, $\mathrm{Na}(\alpha-\mathrm{ISA})$ or degradation solutions were added. The final concentration of $\mathrm{Th}\left({ }^{232} \mathrm{Th}\right)$ in the ACW-I was approximately $6.7 \cdot 10^{-9} \mathrm{M}$. The suspensions were further equilibrated for $24 \mathrm{~h}$ and then centrifuged at $27,000 \mathrm{~g}$ for 15 minutes. $10 \mathrm{ml}$ of the supernatant were sampled and put in a $20 \mathrm{ml}$ counting vial. The coloured substances of the samples, causing quenching, were oxidised as described for the case with ${ }^{63} \mathrm{Ni}$. The activity of ${ }^{234} \mathrm{Th}$ was measured by liquid scintillation counting using the Cerenkov radiation caused mainly by the daughter radioisotope ${ }^{234} \mathrm{~Pa}$. The centrifuge tubes were emptied, rinsed with demineralised water and refilled with $10 \mathrm{ml}$ of $0.1 \mathrm{M} \mathrm{HCl}$. After shaking the tubes for $2 \mathrm{~h}, 10 \mathrm{ml}$ of the $\mathrm{HCl}$ solution were sampled and analysed for ${ }^{234} \mathrm{Th}$ by liquid scintil- 
lation counting. The sorption coefficient $\left(\mathrm{K}_{\mathrm{d}}\right)$ of ${ }^{234} \mathrm{Th}$ was calculated using the following equation:

$$
\mathrm{K}_{\mathrm{d}}=\frac{\left(\mathrm{A}_{\text {input }}-\mathrm{A}_{\text {wall }}-\mathrm{A}_{\mathrm{eq}} \cdot \mathrm{V}\right)}{\mathrm{A}_{\mathrm{eq}} \cdot \mathrm{W}}
$$

where:

$\mathrm{A}_{\text {input }}=$ activity of the radioisotope added to the system (cpm)

$A_{\text {wall }}=$ activity of the radioisotope sorbed on the vessel wall (cpm)

$\mathrm{A}_{\text {eq }}=$ activity of the radioisotope in the equilibrium solution $\left(\mathrm{cpm} \cdot \mathrm{1}^{-1}\right)$.

A similar procedure to that used for Eu(III) was not possible because of light scattering by the suspended particles. As a consequence of this, mass balance could not be checked and is an assumption used to calculate the distribution coefficient in the case of Th(IV).

\section{Sorption experiments with $\mathrm{Ni}(\mathrm{II})^{2}$}

$500 \mathrm{mg}$ of feldspar were placed in $50 \mathrm{ml}$ polyallomer centrifuge tubes and contacted with $30 \mathrm{ml}$ of either ACW-I, $\mathrm{Na}(\alpha$-ISA) or degradation products and $1 \mathrm{ml}$ ${ }^{63} \mathrm{Ni}\left(222 \mathrm{~Bq} \cdot \mathrm{ml}^{-1}\right.$ in $\left.0.001 \mathrm{M} \mathrm{HCl}\right)$. The total added concentration of $\mathrm{Ni}$ in the ACW-I was approximately $3 \cdot 10^{-10} \mathrm{M}$. The suspensions were equilibrated for $24 \mathrm{~h}$ and then centrifuged at $27,000 \mathrm{~g}$ for 15 minutes. $8 \mathrm{ml}$ of the supernatant were sampled and put in a $20 \mathrm{ml}$ counting vial. The degradation solutions were coloured, which caused quenching in the activity measurements. The coloured substances in the samples were oxidised by adding $100 \mu \mathrm{l}$ of $30 \% \mathrm{H}_{2} \mathrm{O}_{2}$ to the solutions. The solutions were left standing overnight, heated at $80^{\circ} \mathrm{C}$ for one hour and finally $2 \mathrm{ml}$ of $6 \mathrm{M}$ $\mathrm{HCl}$ were added. After this treatment the solutions were virtually colourless. The activity of ${ }^{63} \mathrm{Ni}$ was measured by liquid scintillation counting (Tricarb $^{\circledast}$ $2250 \mathrm{CA}$, Packard), using $10 \mathrm{ml}$ Instagel ${ }^{\otimes}$ (Packard) as the scintillation cocktail. The centrifuge tubes were emptied, rinsed with demineralised water and refilled with $10 \mathrm{ml}$ of $0.1 \mathrm{M} \mathrm{HCl}$ to remove the $\mathrm{Ni}$ sorbed on the vessel walls. After shaking the tubes for $2 \mathrm{~h}, 10 \mathrm{ml}$ of the $\mathrm{HCl}$ solution were sampled, $10 \mathrm{ml}$ Instagel added and analysed for ${ }^{63} \mathrm{Ni}$ by liquid scintillation counting. The sorption coefficient $\left(\mathrm{K}_{\mathrm{d}}\right)$ of Ni was calculated using Eq. (2) assuming $100 \%$ mass balance.

\section{Uncertainty estimation}

The observed scatter in the data could not be satisfactorily described by statistical propagation of the errors involved in scintillation counting and other experimental procedures. Hence, unknown error sources such as material inhomogeneities seem to be responsible for this discrepancy. For this reason, the observed

\footnotetext{
${ }^{2}$ Note that for practical reasons, a similar method as used for $\mathrm{Eu}$ and Th could not be applied for $\mathrm{Ni}$.
}

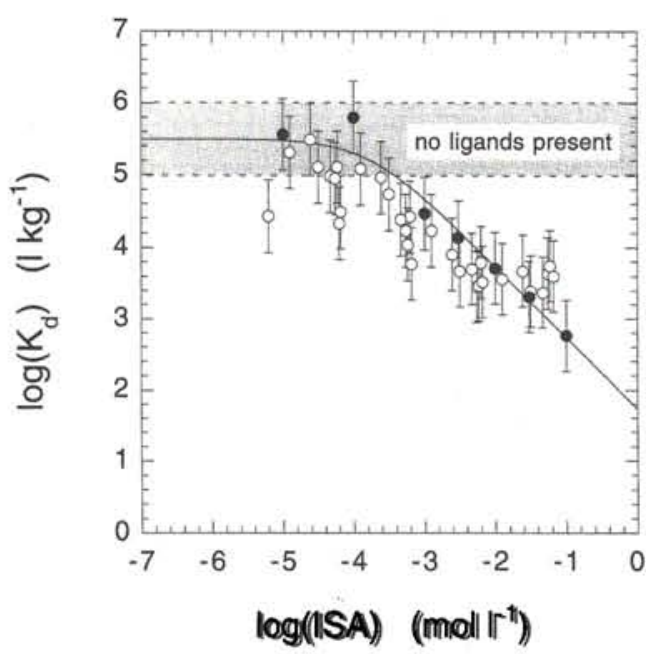

Fig. 1. Dependence of the sorption of Eu(III) on feldspar at $\mathrm{pH}=13.3$ on the concentration of $\alpha$-ISA in pure $\alpha$-ISA solutions (closed symbols) and in solutions containing Aldrich cellulose degradation products (open symbols). The solid line represents the case for the formation of a $1: 1$ complex between $\mathrm{Eu}(\mathrm{III})$ and $\alpha$-ISA.

standard deviation of replicate measurements was used to describe the uncertainty of the data. The largest uncertainty was observed for $K_{d}$ values measured in absence of organic ligands due to the low activity in the equilibrium solution. This value was assumed to be valid for all the other experimental conditions, which rather overestimates the effective errors.

\section{Results and discussion}

Fig. 1 shows the effect of $\alpha$-ISA and degradation products of the Aldrich cellulose on the sorption of Eu(III) on feldspar in ACW-I. The effect of the cellulose degradation products is plotted as a function of the $\alpha$-ISA concentration in the degradation mixtures. Note that the shaded area in the figures represents measurements of $K_{d}$ in absence of ligands $\left(K_{d}=K_{d}{ }^{0}\right)$. Both pure $\alpha$-ISA and the degradation products have a reducing effect on the sorption of Eu(III). The good agreement between the reduction caused by pure $\alpha$-ISA and by the degradation products of Aldrich cellulose indicates that $\alpha$-ISA in the mixture of these cellulose degradation products is mainly responsible for the reducing effect. The effect may be, by analogy with gluconic acid, interpreted to be caused by a strong complexation between Eu(III) and $\alpha$-ISA. According to a generalised coordination scheme for the complexation of metals with polyhydroxy carboxylic acids [5], the complexation of a metal $\mathrm{M}$ with ISA can be written as:

$$
\begin{aligned}
& \mathrm{M}^{2+}+\mathrm{nH}_{4} \mathrm{ISA}^{-}+\mathrm{mH}_{2} \mathrm{O} \Leftrightarrow \\
& \quad \mathrm{M}(\mathrm{OH})_{\mathrm{m}}\left(\mathrm{H}_{4-\mathrm{p}} \mathrm{ISA}\right)_{\mathrm{n}}^{2-(m+n p+n)}+(\mathrm{m}+\mathrm{np}) \mathrm{H}^{+} .
\end{aligned}
$$

$\mathrm{H}_{4} \mathrm{SSA}^{-}$in Eq. (3) corresponds to the $\alpha$-ISA used throughout the text. " $\mathrm{H}_{4}$ " represents the protons of the 
Table 1. Overview of the fit parameters used for fitting data in Figs. 1, 2, 4 and 6. Only $K_{M}$ is a fit parameter. The other parameters, $\mathrm{n}$ and $\mathrm{K}_{\mathrm{d}}{ }^{\circ}$, were fixed.

\begin{tabular}{llllcc}
\hline $\mathrm{M}$ & Data & Ligand & $\mathrm{n}$ & $\log \mathrm{K}_{\mathrm{d}}^{0}$ & $\mathrm{~K}_{\mathrm{M}}\left(\mathrm{l}^{\mathrm{n}} \cdot \mathrm{md}^{-\mathrm{n}}\right)$ \\
\hline Eu(III) & Fig. 1 & $\alpha$-ISA & 1 & 5.5 & $(5.8 \pm 1.4) \cdot 10^{3}$ \\
Eu(III) & Fig. 2 & $\alpha$-ISA & 1 & 5.5 & $(10.3 \pm 3.5) \cdot 10^{3}$ \\
Eu(III) & Fig. 2 & $\beta$-ISA & 1 & 5.5 & $93 \pm 74$ \\
Th(IV) & Fig. 4 & $\alpha$-ISA & 2 & 5.2 & $(7.7 \pm 5.6) \cdot 10^{7}$ \\
Ni(II) & Fig. 6 & $\alpha$-ISA & 1 & 1.8 & $31 \pm 9$ \\
\hline
\end{tabular}

4 hydroxy-groups in $\alpha$-ISA. Note that the protons arising in the right hand side of reaction (3) originate from the deprotonation of hydroxyl groups of ISA and of coordinated water. The stability constant is defined by:

$\mathrm{K}_{\text {MHISA }}=\frac{\left[\mathrm{M}(\mathrm{OH})_{\mathrm{m}}\left(\mathrm{H}_{4-\mathrm{p}} \mathrm{ISA}\right)_{\mathrm{n}}{ }^{2-(\mathrm{m}+\mathrm{np}+\mathrm{n})}\right] \cdot\left[\mathrm{H}^{+}\right]^{\mathrm{m}+\mathrm{np}}}{\left[\mathrm{M}^{\mathrm{z}}\right] \cdot\left[\mathrm{H}_{4} \mathrm{ISA}^{-}\right]^{\mathrm{n}}}$

where the terms in parenthesis represent concentrations of the species $\left(\mathrm{mol} \cdot \mathrm{1}^{-1}\right)$. The effect of ISA on the sorption $\left(\mathrm{K}_{\mathrm{d}}\right)$ of a metal $\mathrm{M}$ on a solid phase $\mathrm{S}$ can be described by $[17,18]$ :

$$
\mathrm{K}_{\mathrm{d}}=\frac{\mathrm{K}_{\mathrm{d}}{ }^{0}}{1+\left(\frac{\mathrm{K}_{\mathrm{MHISA}} \cdot\left[\mathrm{H}_{4} I S A^{-}\right]^{\mathrm{n}}}{\left[\mathrm{H}^{+}\right]^{\mathrm{m}+\mathrm{np}} \cdot \mathrm{A}}\right)}
$$

where:

$$
\mathrm{A}=1+\sum_{\mathrm{i}=1}^{\mathrm{n}} \beta_{\mathrm{i}}^{\mathrm{OH}} \cdot[\mathrm{OH}]^{\mathrm{i}}
$$

with:

$\mathrm{K}_{\mathrm{d}}=$ distribution coefficient of $\mathrm{M}^{2+}$ in presence of ISA

$\mathrm{K}_{\mathrm{d}}{ }^{0}=$ distribution coefficient $\mathrm{M}^{2+}$ in absence of ISA $\beta_{i}^{\mathrm{OH}}=$ the hydrolysis constants of $\mathrm{M}^{2+}$.

Prerequisites for the applicability of Eq. (5) are i) linear and reversible sorption of $\mathrm{M}$ on $\mathrm{S}$, and ii) no sorption of the complexes.

At a given $\mathrm{pH},\left[\mathrm{H}^{+}\right]$and $\mathrm{A}$ are constant and can be combined with $\mathrm{K}_{\mathrm{MHISA}}$ in one conditional constant $\mathrm{K}_{\mathrm{M}}$ :

$$
\mathrm{K}_{\mathrm{M}}=\frac{\mathrm{K}_{\mathrm{MHISA}}}{\left[\mathrm{H}^{+}\right]^{\mathrm{m}+\mathrm{np}} \cdot \mathrm{A}}
$$

Combining Eqs. (5) and (7) gives:

$$
\mathrm{K}_{\mathrm{d}}=\frac{\mathrm{K}_{\mathrm{d}}{ }^{0}}{1+\mathrm{K}_{\mathrm{M}} \cdot\left[\mathrm{H}_{4} \mathrm{ISA}^{-}\right]^{\mathrm{n}}}
$$

In a first approach, the data for $\mathrm{Eu}(\mathrm{III})$ were analysed assuming that reaction (3) takes place and that the prerequisites for Eq. (5) were fulfilled. The solid line in Fig. 1 represents a least squares fit to the pure $\alpha$-ISA data, using Eq. (8) with $K_{M}$ as a fit parameter. It was assumed that $\mathrm{Eu}$ forms a 1:1 complex with $\alpha$-ISA $(n=1)$. The value of the fit parameter $K_{M}$ and of the other parameters used, i.e. $\mathrm{n}$ and $\mathrm{K}_{\mathrm{d}}{ }^{0}$, are summarised in Table 1 . The data are fitted well by Eq. (8), indicat-

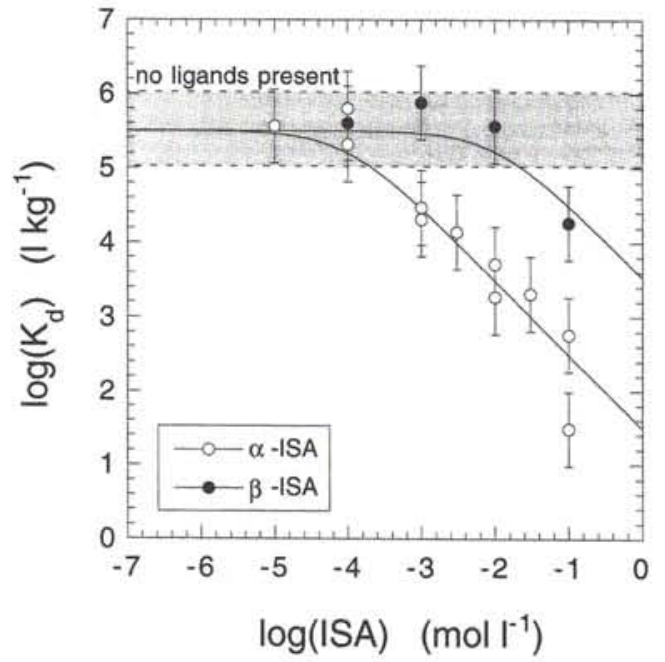

Fig. 2. Effect of pure $\alpha$-ISA and $\beta$-ISA on the sorption of $\mathrm{Eu}$ (III) on feldspar at $\mathrm{pH}=13.3$. The data were measured in experiments with a reduced volume. The solid lines represent the case for the formation of a $1: 1$ complex between Eu(III) and $\alpha$-ISA or $\mathrm{Eu}(\mathrm{III})$ and $\beta$-ISA.

ing that the assumptions made, i.e. reversible sorption of $\mathrm{Eu}$, formation of a 1:1 complex and no sorption of Eu-ISA, appear to be justified for the given situation. In principle it is possible to calculate a complexation constant from the conditional constant $\mathrm{K}_{\mathrm{M}}$, but as the complexation reaction is not known - neither are the values of $m$ and of $p$ - no benefit can be derived from such a calculation. Wieland et al. [19] studied the effect of $\alpha$-ISA on the sorption of Eu(III) on hardened cement paste. They found a negligible effect of $\alpha$-ISA on the sorption of $\mathrm{Eu}(\mathrm{III})$ in the concentration range $10^{-5} \mathrm{M}<[\alpha-$ ISA $]<10^{-2} \mathrm{M}$. Only for $[\alpha-$-ISA] $>$ $10^{-2} \mathrm{M}$ was a significant effect observed. The difference between the effects observed by Wieland $e t$ al. [19] and those described in this study cannot be explained properly. One possibility is that Eu-ISA complexes could sorb on the cement. This hypothesis is based on the observation that $\alpha$-ISA sorbs strongly on hydrated cement under alkaline conditions [20] ( $\alpha$-ISA does not sorb on feldspar [13]).

Fig. 2 shows the effect of pure $\alpha$-ISA and $\beta$-ISA on the sorption of $\mathrm{Eu}(\mathrm{III})$ on feldspar as measured in the experiments with a reduced volume. The effect of $\beta$-ISA on the sorption of Eu(III) is significantly lower than that of $\alpha$-ISA. This can be explained by a differ- 


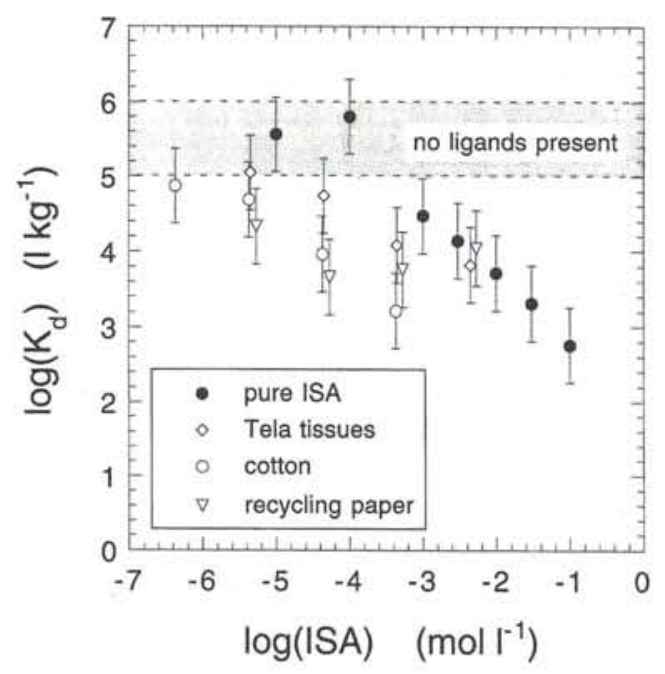

Fig. 3. Dependence of the sorption of Eu(III) on feldspar at $\mathrm{pH}=13.3$ on the concentration of $\alpha$-ISA in pure $\alpha$-ISA solutions and solutions containing degradation products of Tela tissues, cotton and paper.

ence in complexation behaviour: $\beta$-ISA seems to form less stable complexes with $\mathrm{Eu}(\mathrm{III})$ than $\alpha$-ISA. The data in Fig. 2 were fitted using Eq. (8) with a constant $\mathrm{K}_{\mathrm{M}}$ for the Eu- $\beta$-ISA complex roughly two orders of magnitude lower than the one of the Eu- $\alpha$-ISA complex (see Table 1). As $K_{M}$ is proportional to the stability of the complex (see Eq. (7)) this means that the stability of the complex between Eu(III) and $\beta$-ISA is roughly 2 orders of magnitude lower, assuming that a similar complexation reaction (Eq. (3)) takes place. As both isomers are present in equal amounts in the solution of degraded cellulose [2], the speciation of Eu(III) in solutions of degraded cellulose will be dominated by the Eu- $\alpha$-ISA complex. This explains why the effect of cellulose degradation products and the effect of pure $\alpha$-ISA on the sorption of $\mathrm{Eu}(\mathrm{III})$ are very similar.

Fig. 3 shows the effect of pure $\alpha$-ISA and of degradation products of other cellulosic materials (Tela tissues, cotton and recycling paper) on the sorption of $\mathrm{Eu}(\mathrm{III})$. A larger effect on the sorption of Eu(III) on feldspar can be observed for the degradation products than for the pure $\alpha$-ISA. This effect can only be explained by the presence of other ligands than $\alpha$-ISA. It was shown elsewhere [2] that cellulose contains, beside pure cellulose, also non-cellulose compounds that are readily soluble in alkaline solutions. These unknown compounds might have - in addition to the effect of $\alpha$-ISA - a small effect on the sorption of radionuclides.

Fig. 4 shows the effect of pure $\alpha$-ISA and Aldrich cellulose degradation products on the sorption of Th(IV) on feldspar. It is clear that $\alpha$-ISA and the cellulose degradation products have an adverse effect on the sorption of Th(IV). If the data of the degradation products are normalised with respect to the $\alpha$-ISA concentration, the agreement between pure $\alpha$-ISA and the cellulose degradation products is relatively good. Consequently, the effect of the cellulose degradation

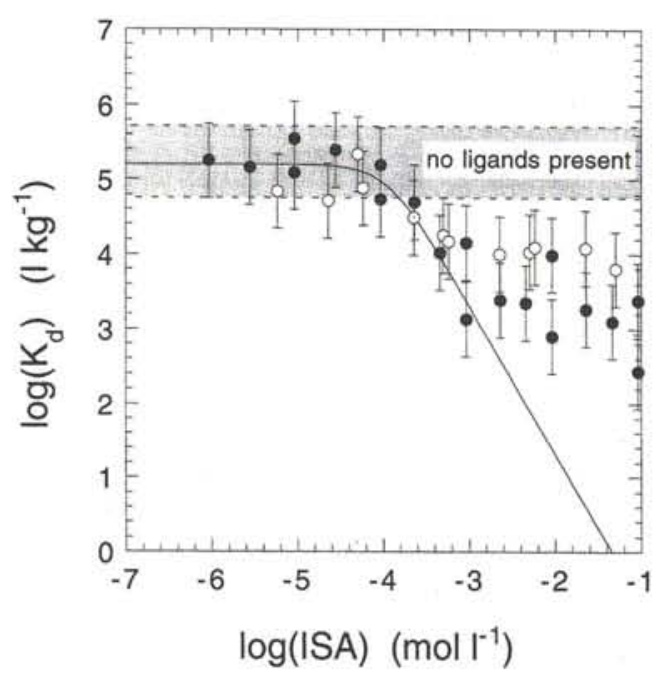

Fig. 4. Effect of cellulose degradation products (open symbols: Aldrich, degradation time 1 year) and $\alpha$-ISA (closed symbols) on the sorption of $\mathrm{Th}(\mathrm{IV})$ on feldspar at $\mathrm{pH}=13.3$. The solid line represents the case for the formation of a 1:2 complex between $\operatorname{Th}(\mathrm{IV})$ and $\alpha$-ISA.

products on the sorption of Th(IV) can be essentially explained by the presence of $\alpha$-ISA. Wieland et al. [19] studied the sorption of Th(IV) on hardened cement past under similar conditions and found a significant effect of $\alpha$-ISA on the sorption of Th(IV) in the range $10^{-5} \mathrm{M}<[\alpha-$ ISA $]<10^{-2} \mathrm{M}$. The data could be explained by the formation of a $1: 2$ Th-ISA complex. The formation of a 1:2 complex is in good agreement with recent observations that in presence of $\mathrm{Ca}(\mathrm{II})$, ISA forms 1: 2 complexes with Th(IV), Ca being part of the complex [21] and with studies of the coordination between $\mathrm{Al}$ (III), aldarate ligands and $\mathrm{Ca}$ (II) [22]. In the absence of $\mathrm{Ca}$ (II), a 1:1 complex between Th(IV) and $\alpha$-ISA is formed [21]. Based on these findings, and because ACW-I contains $\sim 2 \mathrm{mM} \mathrm{Ca}$ (II), the data for pure $\alpha$-ISA were analysed assuming that a $1: 2$ complex between Th and $\alpha$-ISA is formed $(n=2)$. The solid line in Fig. 4 is a least square fit of the $\alpha$-ISA data using Eq. (8) with $\mathrm{K}_{\mathrm{M}}$ as a fit parameter. The fit parameter is given in Table 1 . As can be seen, the data can be fitted satisfactorily by Eq. (8) for $10^{-6} \mathrm{M}<$ [ISA] $<10^{-3} \mathrm{M}$. Beyond a concentration of $10^{-3} \mathrm{M}$, the effect of ISA on the sorption becomes weaker. This might be an indication of a partially irreversible sorption of Th(IV). As a consequence, Eq. (8) must be extended with irreversible sorption in order to describe our data. This, however, is far beyond the scope of this work. Moreover, as long as there are no more evidences for such an irreversible sorption of Th(IV), we see no benefit from modelling our data with partially irreversible sorption at the moment. A more detailed experimental programme on reversible/irreversible sorption is required to test this hypothesis.

Fig. 5 shows the effect of pure $\alpha$-ISA and degradation products of other cellulosic materials (Tela tissues, cotton and recycling paper) on the sorption of Th(IV). Also for the other cellulosic materials, $\alpha$-ISA 


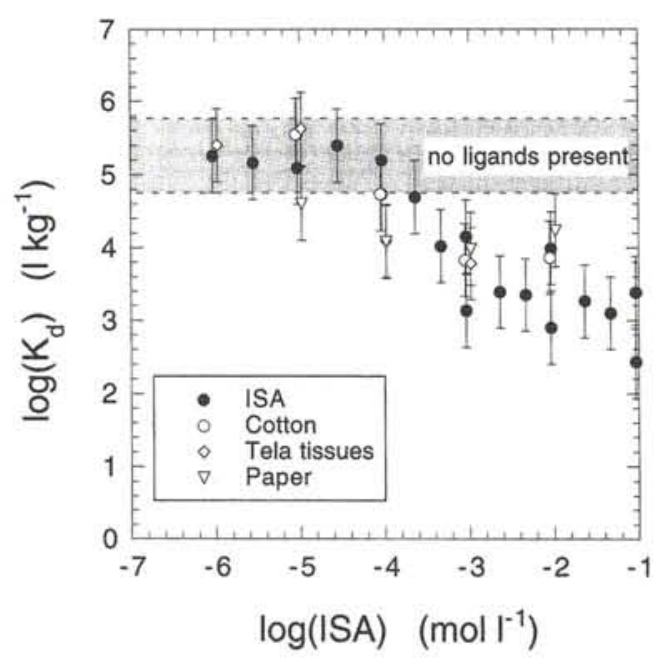

Fig. 5. Effect of cellulose degradation products (open symbols: Tela, cotton, paper: degradation time 1 year) and $\alpha$-ISA (closed symbols) on the sorption of $\mathrm{Th}(\mathrm{IV})$ on feldspar at $\mathrm{pH}=13.3$.

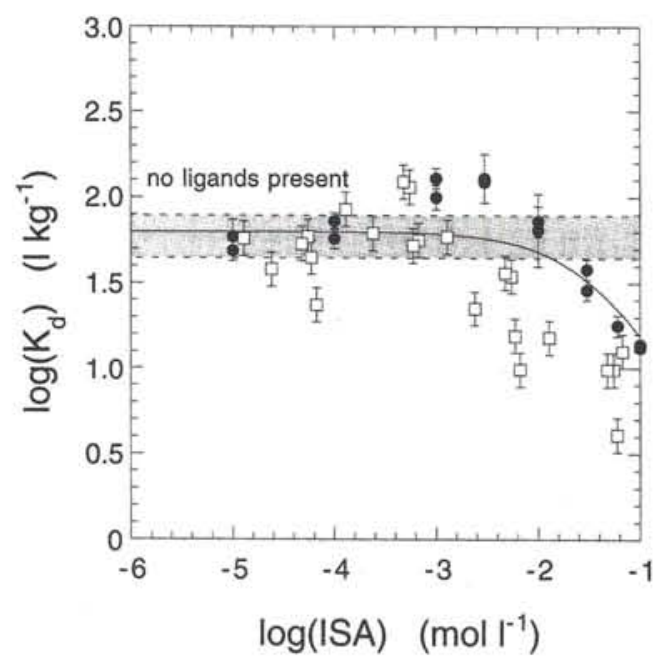

Fig. 6. Dependence of the sorption of $\mathrm{Ni}(\mathrm{II})$ on feldspar at $\mathrm{pH}=$ 13.3 on the concentration of $\alpha$-ISA for pure $\alpha$-ISA solutions (closed symbols) and solutions containing degradation products of Aldrich cellulose (open symbols). The solid line represents the case for the formation of a 1:1 complex between $\mathrm{Ni}(\mathrm{II})$ and $a$-ISA.

is the dominant ligand and explains principally the observed sorption reduction. Unlike Eu-sorption, the Thsorption is not affected by the readily soluble organics present in cellulosic materials.

Fig. 6 shows the effect of $\alpha$-ISA and Aldrich cellulose degradation products on the sorption of $\mathrm{Ni}$ (II) on feldspar. Also in the case of $\mathrm{Ni}(\mathrm{II})$, ISA and cellulose degradation products have an adverse effect on the sorption, indicating that a complex between $\alpha$-ISA or cellulose degradation products, and $\mathrm{Ni}$ (II) is formed. The data for the degradation products are normalized with respect to $\alpha$-ISA. The agreement between pure $\alpha$-ISA and the Aldrich cellulose degradation products is less good. The degradation products tend to have a larger effect on sorption, probably due to the presence of other organics as was discussed earlier for $\mathrm{Eu}(\mathrm{III})$.

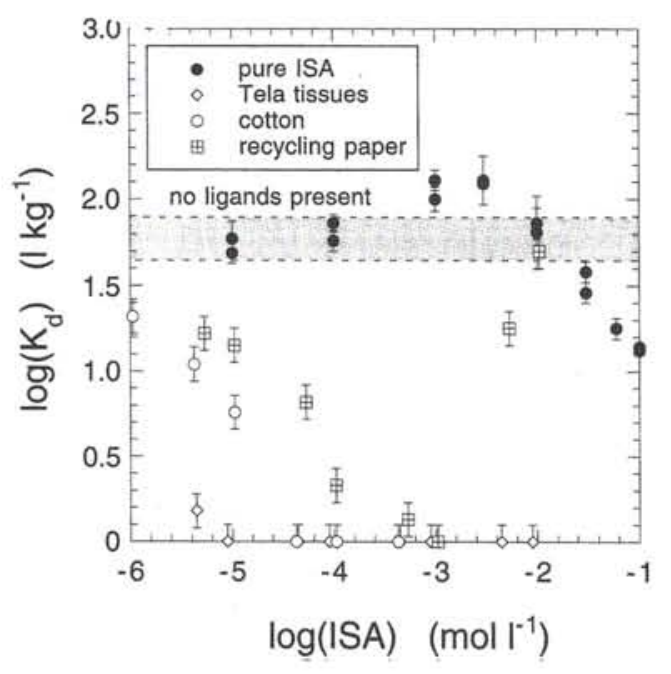

Fig. 7. Dependence of the sorption of $\mathrm{Ni}$ (II) on feldspar at $\mathrm{pH}=$ 13.3 on the concentration of $\alpha$-ISA for pure $\alpha$-ISA solutions (closed symbols) and solutions containing degradation products of cotton, Tela tissues and recycling paper (open symbols).

The data for pure $\alpha$-ISA have been fitted (solid line in Fig. 6) using Eq. (8) with $n=1$ (formation of a $1: 1$ complex between $\mathrm{Ni}(\mathrm{II})$ and $\alpha$-ISA). The parameters are given in Table 1 . The data can be fitted reasonably well with Eq. (8) for $[\alpha-I S A]>5 \cdot 10^{-3} \mathrm{M}$. In the concentration range $10^{-4} \mathrm{M}<[\alpha$-ISA $]<5 \cdot 10^{-3} \mathrm{M}$, the sorption of $\mathrm{Ni}(\mathrm{II})$ increases with increasing concentration of $\alpha$-ISA in solution. Such a phenomenon can only be explained by the sorption of Ni-ISA complexes. However, this is in contradiction with the observation that $\alpha$-ISA itself does not sorb on feldspar [13]. More detailed studies are required to obtain more evidence for the sorption of metal complexes.

The degradation products of the other cellulosic materials (cotton, Tela and paper) have a much stronger effect on the sorption of $\mathrm{Ni}$ on feldspar than $\alpha$-ISA (Fig. 7). These effects indicate the presence of other organics which form much stronger complexes with $\mathrm{Ni}$ (II) than $\alpha$-ISA does. The large effect observed for $\mathrm{Ni}$ (II) and the relatively small effect observed for $\mathrm{Eu}$ (III) might indicate that the unknown ligands are polarisable or "soft" ligands [23]. Further investigations are underway to identify the nature of these compounds.

\section{Conclusions}

Degradation products formed by alkaline degradation of different cellulosic materials (Aldrich cellulose, Tela tissues, cotton and paper) have an adverse effect on the sorption of $\mathrm{Eu}$ (III), Th(IV) and $\mathrm{Ni}$ (II) on feldspar at a pH of 13.3. The effect can be explained by complexation of these metals with the degradation products and with readily soluble ligands present in the cellulosic materials.

For Th(IV), $\alpha$-ISA is the main ligand causing the observed effects in the case of the four cellulosic materials studied. For $\mathrm{Eu}(\mathrm{III}), \alpha$-ISA is the effective ligand 
only in the case of pure Aldrich cellulose. For the other cellulosic materials, other ligands than $\alpha$-ISA seem to cause the observed sorption reduction. The concentration of $\alpha$-ISA in solution has to be larger than $10^{-4}-10^{-3} \mathrm{M}$ in order to cause a significant reduction in the sorption of these metals. The decrease in the sorption of Eu(III) by $\alpha$-ISA can be described by the formation of a 1:1 complex with $\alpha$-ISA, assuming that the metal sorbs reversibly and that the complexes with $a$-ISA do not sorb. In the case of Th(IV), indications for the formation of a 1:2 Th-ISA complex were found. Moreover, part of the Th(IV) seems to sorb irreversibly on the feldspar under alkaline conditions.

For $\mathrm{Ni}(\mathrm{II})$, other ligands than $\alpha$-ISA cause the observed sorption reduction. This could be observed for all cellulosic materials studied. The largest effects could be observed for paper, cotton and Tela tissues. In the case of Aldrich cellulose, the observed effect is only slightly stronger than the effect of pure $\alpha$-ISA. The nature of the other ligands is unknown.

\section{Acknowledgements}

This work was partially financed by the National Cooperative for the Disposal of Radioactive Waste (Nagra). Dr. M. Bradbury (PSI) and Dr. I. Hagenlocher (Nagra) are warmly thanked for giving useful suggestions to improve the manuscript.

\section{References}

1. Glaus, M. A., Van Loon, L. R., Achatz, S., Chodura, A., Fischer, K.: Degradation of Cellulosic Materials under the Alkaline Conditions of a Cementitious Repository for Lowand Intermediate Level Radioactive Waste. Part I: Identification of Degradation Products. Anal. Chim. Acta 398, 111 (1999).

2. Van Loon, L. R., Glaus, M. A., Stallone, S., Laube, A.: Degradation of Cellulosic Materials under the Alkaline Conditions of a Cementitious Repository for Low- and Intermediate Level Radioactive Waste. Part II: Degradation Kinetics. J. Environ. Polym. Degrad. 7, 41 (1999).

3. Greenfield, B. F., Holtom, G. F., Hurdus, M. H., O'Kelly, N., Pilkington, N. J., Rosevear, A., Spindler, M. W., Williams, S. J.: The Identification and Degradation of Isosaccharinic Acid, a Cellulose Degradation Product. Mat. Res. Soc. Symp. Proc. 353, 1151 (1995).

4. Sawyer, D. T.: Metal-Gluconate Complexes. Chem. Rev. 64, 633 (1964).

5. Van Duin, M., Peters, J. A., Kieboom, A. P. G., Van Bekkum, H.: A General Coordination-Ionization Scheme for Polyhydroxy Carboxylic Acids in Water. Recl. Trav. Chim. Pays-Bas 108, 57 (1989).

6. Vercammen, K, Glaus, M. A., Van Loon, L. R.: The Complexation of $\mathrm{Ca}$ with Isosaccharinic Acid at Alkaline Conditions. Acta Chem. Scandinavica 53, 241 (1999).
7. Greenfield, B. F., Harrison, W. N., Robertson, G. P., Somers, P. J., Spindler, M. W.: Mechanistic Studies on the Alkaline Degradation of Cellulose in Cement. NSS/R272, AEA-D \& R-0219, AEA Technology, Harwell, UK (1993).

8. Moreton, A. D.: Thermodynamic Modelling of the Effect of Hydroxycarboxylic Acids on the Solubility of Plutonium at High pH. Mat. Res. Soc. Symp. Proc. 294, 753 (1993).

9. Bourbon, X., Toulhoat, P.: Influence of Organic Degradation Products on the Solubilisation of Radionuclides in Intermediate and Low Level Radioactive Wastes. Radiochim. Acta 74, 315 (1996).

10. Berry, J. A., Bond, K. A., Ferguson, D. R., Pilkington, N. J.: Experimental Studies of the Effects of Organic Materials on the Sorption of Uranium and Plutonium. Radiochim. Acta 52/53, 201 (1991).

11. Baston, G. M. N., Berry, J. A., Bond, K. A., Brownsword, M., Linklater, C. M.: Effect of Organic Degradation Products on the Sorption of Actinides. Radiochim. Acta 58/59, 349 (1992).

12. Baston, G. M. N., Berry, J. A., Bond, K. A., Boult, K. A., Linklater, C. M.: Effects of Cellulosic Degradation Products Concentration on Actinide Sorption on Tuffs from the Borrowdale Volcanic Group, Sellafield, Cumbria. Radiochim. Acta 66/67, 521 (1994).

13. Van Loon, L. R., Glaus, M. A.: Unpublished results (1998).

14. Berner, U.: A Thermodynamic Description of the Evolution of Pore Water Chemistry and Uranium Speciation during the Degradation of Cement. PSI-Bericht 62, Paul Scherrer Institute, Villigen, Switzerland. Also published as Nagra Technical Report series NTB 90-12, Nagra, Wettingen, Switzerland (1990).

15. Rocklin, R. D., Clarke, A. P., Weitzhandler, M.: Improved Long-Term Reproducibility for Pulsed Amperometric Detection of Carbohydrates via a New Quadruple-Potential Waveform. Anal. Chem. 70, 1496 (1998).

16. Dyrssen, D.: The Preparation of Carrier-free Thorium-234 (UX1) by Ion Exchange. Svensk Kemisk Tidskrift 62, 153 (1950).

17. Van Loon, L. R., Kopajtic, $\mathrm{Z}$.: Complexation of $\mathrm{Cu}^{2+}, \mathrm{Ni}^{2+}$ and $\mathrm{UO}_{2}{ }^{2+}$ by Radiolytic Degradation Products of Bitumen. Radiochim. Acta 54, 193 (1991).

18. Van Loon, L. R., Glaus, M. A.: Experimental and Theoretical Studies on Alkaline Degradation of Cellulose and its Impact on the Sorption of Radionuclides. PSI-Bericht 98-07, Paul Scherrer Institute, Villigen, Switzerland. Also published as Nagra Technical Report series NTB 97-04, Nagra, Wettingen, Switzerland (1998).

19. Wieland, E., Tits, J., Spieler, P., Dobler, J. P.: Interaction of Eu(III) and Th(IV) with Sulphate-resisting Portland Cement. Mat. Res. Soc. Symp. Proc. 506, 573 (1998).

20. Van Loon, L. R., Glaus, M. A., Stallone, S., Laube, A.: Sorption of Isosaccharinic Acid, a Cellulose Degradation Product, on Cement. Environ. Sci. Technol. 31, 1243 (1997).

21. Vercammen, K., Glaus, M. A., Van Loon, L. R.: Evidence for the Existence of Complexes between Th(IV) and $\alpha$-Isosaccharinic Acid under Alkaline Conditions. Radiochim. Acta 84, 221 (1999).

22. Venema, F. R., Peters, J. A., Van Bekkum, H.: Multinuclearmagnetic-resonance study of the coordination of aluminium(III)-aldarate complexes with calcium(II) in aqueous solution. Recl. Trav. Chim. Pays-Bas 112, 445 (1993).

23. Pearson, R. G.: Hard and Soft Acids and Bases. J. Am. Chem. Soc. 85, 3533 (1963). 
Brought to you by | Lib4RI Eawag-Empa 